\title{
Semmelweis y su aporte científico a la medicina: Un lavado de manos salva vidas
}

\author{
Marcelo Miranda C. y Luz Navarrete T.
}

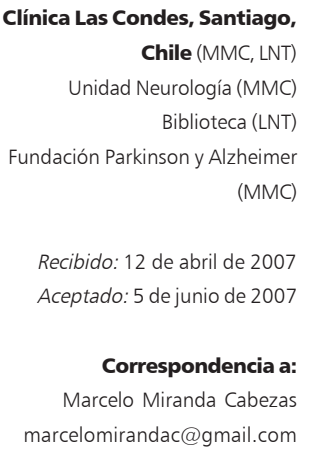

\section{Semmelweis and his outstanding contribution to Medicine: Washing hands saves lives}

Ignaz Semmelweis was a Hungarian obstetrician who in the nineteenth century, preceding the discoveries of Pasteur and Lister, proposed the infectious etiology of puerperal sepsis. With a simple antiseptic procedure, he achieved marked reduction of the prevalence of this disease. However, he needed to fight against the reluctancy of his colleagues who didn't accept his observations although they were for the first time in the history of Science, supported by statistical significance analysis. This report describes biographical data of this revolutionary physician and the circumstances of his strange death based on information not often revealed.

Key words: Semmelweis, IP; puerperal infections; antisepsis.

Palabras clave: Semmelweis IP; sepsis puerperal; antisepsis.

\section{$\mathrm{L}$} a sepsis ocasiona en el mundo 1.400 muertes cada día, muchos de estos pacientes adquieren la infección estando hospitalizados y constituye la complicación intra-hospitalaria más frecuente ${ }^{1}$. La infección nosocomial de pacientes por las manos contaminadas del personal de salud es una de la formas de diseminación de los agentes infecciosos. En la actualidad, la higiene de las manos es el factor individual más importante para el control de las infecciones. Aún en nuestros días, el lavado de manos es realizado sólo un tercio a la mitad de lo frecuente que debería ser hecho ${ }^{1}$. En la actualidad, es difícil entender que un hecho tan rutinario en la práctica de la medicina, como lavarse las manos previo a examinar a un paciente, haya causado tanta controversia e, incluso, oprobio a la persona que lo planteó como una medida básica para la atención de un enfermo. Ese fue el caso de Ignaz Semmelweis quién no sólo descubrió que esta simple medida salvaba vidas, sino que por primera vez aplicó la comprobación estadística a sus hallazgos.

Esta comunicación revisa algunos aspectos de su vida y los hechos que llevaron a uno de los descubrimientos que han cambiado la historia de la medicina.

Datos biográficos. Ignaz Philipp Semmelweis, nació el 1 de julio de 1818 en Taban, (orilla derecha del río Danubio, capital de Hungría) (Figura 1). Fue el quinto de siete hermanos e hijo de comerciantes. Hungría era entonces parte del imperio austríaco, cuya capital era Viena. Sus estudios médicos los realizó inicialmente en Hungría y luego en Viena, graduándose como médico obstetra en agosto de 1844. En Viena logró amistad con destacados médicos como el anátomo-patólogo Karl von Rokitansky, Josef Skoda y Ferdinand von Hebra. Rokitansky es uno de los fundadores de la anatomía patológica, Skoda de los métodos de auscultación y percusión pulmonar y Hebra es considerado padre de la dermatología $a^{3-7}$.

A los 28 años de edad fue nombrado asistente de la primera clínica ginecológica de Viena. La práctica obstétrica de Semmelweis se inició en el Allgemeines KrankenHaus, el gran hospital general de Viena. La clínica vienesa recibía estudiantes de varias partes de Europa, pero la fiebre puerperal hacía estragos. Semmelweis, muy conmovido por lo que observaba, empezó a recopilar información, a cuantificar datos y reflexionar sobre lo que estudiaba. Comenzó a apreciar diferencias en las frecuencias de presentación de la enfermedad entre las dos salas de maternidad existentes y concluyó, luego de grandes esfuerzos y búsquedas, con la elaboración de un nuevo concepto: existía una "materia cadavérica" que era transportada por las manos de los médicos y estudiantes que tenían a su cargo la atención de las madres en trabajo de parto en la Clínica 1 y generaba en ellas la fatal enfermedad ${ }^{3-7}$. Propuso el uso de soluciones con cloro para el lavado de manos de los médicos, antes y después de atender y examinar a sus pacientes (Figuras 2 y 3 ). Esta medida se inició a mediados de mayo de 1847. Minuciosamente anotó durante temporadas el comportamiento de las muertes y descubrió que, con la medida del lavado de 
manos, éstas disminuyeron extraordinariamente. Consultó los archivos y registros del hospital de maternidad de Viena desde su apertura en 1784 hasta 1848. Elaboró tablas con los datos de partos, defunciones, y tasas de mortalidad para esos años. Registró enormes diferencias en las tasas de mortalidad, por ejemplo, del 12,11\% n 1842 contra el 1,28\% en 1848. Verificó el efecto fatal de la atención obstétrica por parte de los estudiantes de medicina, en comparación con las tasas menores entre las pacientes asignadas a las matronas en la Clínica 2, quienes no tenían contacto con los estudios anatómicos en cadáveres ${ }^{7}$.

Diferentes razones eran dadas para explicar aquella diferencia: la angustia que causaba el sonido de la campanilla del acólito que precedía al sacerdote, cuando éste se dirigía allá para administrar los sacramentos a las moribundas; la vergüenza que sentían las mujeres ante los estudiantes, y la mala ventilación. Semmelweis sabía que esas razones estaban erradas, pero no así cuál era la naturaleza de la fiebre puerperal. El hecho decisivo fue la muerte de su amigo Kolletschka, profesor de medicina legal: al hacer una autopsia un discípulo lo hirió en un dedo ${ }^{3-7}$. Murió con los mismos síntomas que los de la fiebre puerperal, y los hallazgos de su necropsia fueron, en todo, similar a lo hallado en las madres y sus hijos víctimas de la fiebre puerperal. Semmelweis defendió con vigor su descubrimiento y la salud de sus pacientes, Hay que terminar con la matanza, escribió. Concluye "... Una vez que se identificó la causa de la mayor mortalidad de la primera



Figura 1. Retrato de Semmelweis a los 39 años en 1857 en su época de obstetra en Viena ${ }^{2}$. clínica como las partículas de cadáveres adheridas a las manos de los examinadores, fue fácil explicar el motivo por el cual las mujeres que dieron a la luz en la calle tenían una tasa notablemente más baja de mortalidad que las que dieron a luz en la clínica..."2. Postuló que estas partículas cadavéricas entraban por

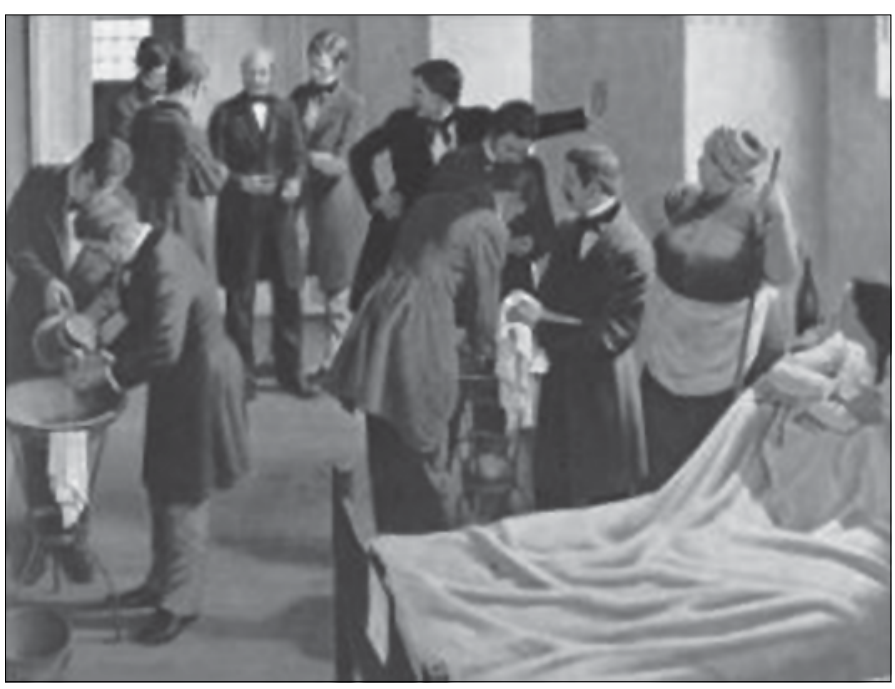

Figura 2. Dibujo ambientado en la época, de una sala de partos del Hospital de Viena representando a Semmelweis enseñando a sus alumnos².

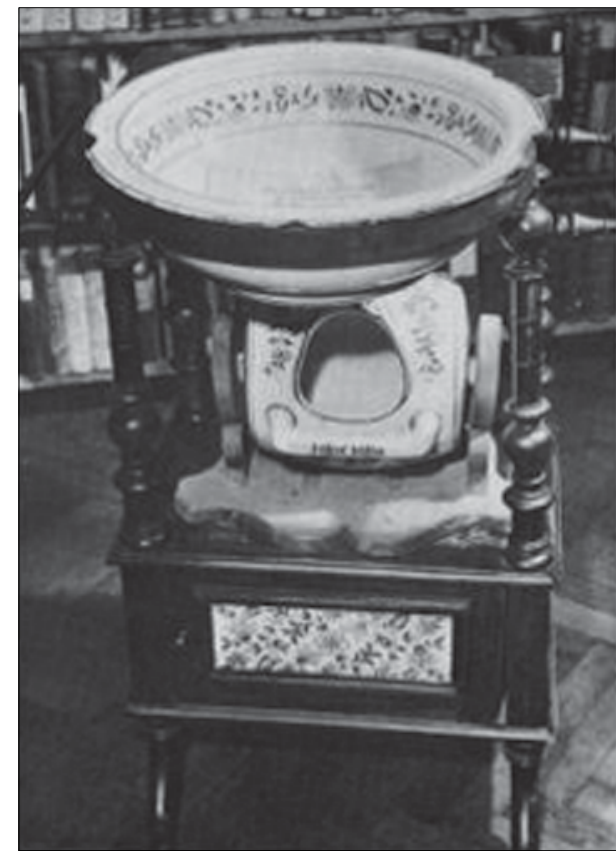

Figura 3. El lavamanos que usó Semmelweis previo al examen de sus pacientes ${ }^{2}$. 
el torrente sanguíneo de la persona afectada y que podía afectar no sólo a puérperas sino a las embarazadas y a sus propios hijos recién nacidos ${ }^{2-6}$. Semmelweis indujo la enfermedad en animales como conejos, pero decidió no utilizar el microscopio para observar los tejidos afectados, ya que lo consideró irrelevante $e^{3,7-11}$. Sus observaciones no tuvieron eco, él mismo fue amenazado. No era posible que se culpara a los propios médicos de estas muertes, era un insulto para la imagen de los médicos. Incluso su propio jefe de Obstetricia, el Profesor Klein, estuvo en contra de él y prohibió esta medida sanitaria, relevando del cargo a Semmelweis en 1849 y dejando a Braun, quien creía que todo era problema de mala ventilación: con lo que la tasa de mortalidad aumentó nuevamente. Lleno de amargura dejó la clínica, asumió la cátedra de Obstetricia Teórica y Práctica en la Universidad de Pest en Hungría, logrando aplicar su método y reduciendo notoriamente la tasa de mortalidad ${ }^{3,7-11}$.

Existían ya antecedentes de que medidas preventivas reducían la fiebre puerperal, así el obstetra escocés Alexander Gordon de Aberdeen, (Escocia), reconoció el carácter epidémico de la condición y recomendó el lavado riguroso de médicos y enfermeras, quemar la ropa de las personas afectadas y fumigación de la ropa de atención de los profesionale ${ }^{7}$. El Dr. Robert Collins, jefe del Hospital Rotunda de Dublín, Irlanda, durante 1829 combatió exitosamente la enfermedad con una exhaustiva limpieza con preparados de cloro en las salas de atención ${ }^{7}$. Las sábanas y ropa se lavaron al seco a temperaturas de 120 a $180{ }^{\circ} \mathrm{C}$. Oliver Wendell Holmes, médico y escritor norteamericano publicó en 1843 un artículo llamado "La contagiosidad de la fiebre puerperal”. Holmes advertía del riesgo de transmisión de miasmas por los propios médicos que habían hecho disecciones y luego atendían partos, validando las medidas preventivas tomadas por Collins en Dublín. Fue rechazado por sus colegas obstetras norteamericanos y sus observaciones no fueron consideradas en su propio país ${ }^{6,7,11}$.

Su propia enfermedad. A partir de 1860, el ánimo de Semmelweis decayó, sufrió importantes episodios depresivos, arranques de irritabilidad y cambios conductuales como la decisión de atender a sus pacientes en Budapest sólo en la noche. Esta sintomatología fue atribuida al estrés y a la incomprensión sufrida por parte de sus pares. Biógrafos como Frank Slaughter en 1950, plantearon que las experiencias trágicas que le tocó vivir a Semmelweis "destruyeron su mente” y lo hicieron "un mártir de la estupidez del mundo", "los largos años de controversia, la amarga frustración sufrida, el recuerdo de las pacientes que vio morir, primero por no poder descubrir porqué morían y luego porque sus colegas no podían enten- der los simples principios que él propuso para evitar las muertes; todas estas cosas fueron cargas demasiado grandes que pueden haber destruido la salud de cualquiera. Su tendencia natural a la tristeza aumentó, hubo días que prácticamente no hablaba a sus colegas, haciendo clases en un lenguaje monótono e incomprensible a sus alumnos interrumpido por arengas que hacía a ratos sin mayor sentido"7.

Sus amigos observaron un progresivo deterioro intelectual, psicosis con rasgos paranoideos y decidieron internarlo en un sanatorio en Viena, a cargo del psiquiatra Riedel en julio de 1865. En el sanatorio se descubrió que Semmelweis presentaba una herida en unos de sus dedos, atribuyéndose a alguna cirugía, pero parece más cierto que se haya provocado en las medidas de contención que necesitó. La herida se gangrenó, se desarrollaron múltiples abscesos y Semmelweis murió el 13 de agosto de 1865 a los 47 años de edad ${ }^{3,7-11}$.

Biógrafos más recientes como Nuland ${ }^{3}$, plantean una visión distinta de los hechos, en el sentido que, Semmelweis, por su especial carácter, haya inducido el rechazo que sufrió. Esto habría sido por su actitud arrogante, descalificadora con los colegas y la demora en publicar en 14 años sus estudios, lo que habría motivado las reticencias en aceptar sus observaciones $^{3}$. Nuland plantea que haya sufrido una enfermedad de Alzheimer como explicación de su demencia, y que muriera a consecuencia de los golpes recibidos para tratar de controlarlo en el sanatorio en que se encontraba internado. Esto, sin embargo, no se apoya en los hallazgos de la necropsia realizada y que fueron revelados recién en $1947^{7,11}$. Estos hallazgos describen múltiples focos sépticos renales, óseos y pulmonares derivados de la lesión gangrenosa del dedo medio derecho, lo que es compatible con lo que ya se conocía. Sin embargo, son muy interesantes además los cambios en el sistema nervioso central, compatibles con una meningitis crónica incluyendo la médula espinal. Para Bauer, estos hallazgos apoyan la presencia de una neurosífilis. Perfectamente esta condición, muy frecuente en esa época, da cuenta de los cambios neuropsiquiátricos y la precoz demencia de Semmelweis ${ }^{7}$.

Su única obra en que resume su experiencia se publicó en 1861: Etiología, concepto y profilaxis de la fiebre puerperal.

Esa época fue muy importante en avances científicos. Rudolf Virchow, ya comenzaba a hablar de higiene y medicina social; sin embargo, Virchow no apoyó a Semmelweis en sus postulados. Años más tarde formularía la tesis "Omnia cellula a cellula" (cada célula surge de otra célula) y llegaría a entender el organismo vivo como una especie de "estado" constituido por 
células. La posterior obra de Virchow "La Patología Celular" sería uno de los fundamentos de la comprensión y el avance de la medicina en lo que restó de la segunda mitad del siglo XIX. En el año de 1848, Claude Bernard en Francia, descubrió en el páncreas de animales una sustancia que emulsionaba y saponificaba las grasas (hoy esta es la enzima lipasa pancreática); el método de la "medicina experimental" fue tomando cuerpo. Más adelantada la segunda mitad del siglo XIX, Pasteur y Koch, aclararían, finalmente, los postulados básicos de la enfermedad como producto de la infección bacteriana y se demostró la existencia del "contagium animatum", los microorganismos. Pasteur reconoció el mérito de Semmelweis en 1879, en una reunión de la Academia de Ciencias de París, en que se discutió sobre la fiebre puerperal. Mientras un expositor hablaba de posibles causas de este cuadro, Pasteur lo interrumpió diciendo: "nada de eso explica la fiebre puerperal: es la enfermera y el médico que llevan los microbios de una persona infectada a otra sana"7. En ese año Pasteur identificó al estreptococo como el agente causal.

Semmelweis es un extraordinario ejemplo de observación metódica, raciocinio y reflexión, es el resultado de uno de los más auténticos casos de compromiso personal con la vocación de médico. Lo que hoy nos parece tan evidente u obvio, representó en su momen- to un cambio de visión asimilado por pocos. Tuvo que avanzar en medio de incomprensiones y de dificultades.

La importancia del aporte de Semmelweis a la obstetricia y medicina en general no ha sido aún superado siquiera por los avances de las nuevas tecnologías genéticas de los últimos años del siglo XX. La historia ha valorado a este médico húngaro de modo justo después de su muerte. Su vida fue la de un hombre que luchó con entereza y sin vacilación por sus ideales y convicciones.

\section{Resumen}

Ignaz Semmelweis, fue un obstetra húngaro que a mediados del siglo XIX, precediendo los hallazgos de Pasteur y Lister, logró descubrir la naturaleza infecciosa de la fiebre puerperal, logrando controlar su aparición con una simple medida de antisepsia. Debió luchar con la reticencia de sus colegas que no aceptaron sus observaciones que, por primera vez en la historia, fueron apoyadas con datos estadísticos. Esta comunicación describe datos biográficos de este trascendente científico y las circunstancias que rodearon su extraña muerte, apoyada en información infrecuentemente divulgada.

\section{Referencias}

1.- Best M, Neuhauser D. Ignaz Semmelweis and the birth of infection control. Qual Saf Health Care 2004; 13: 233-4.

2.- http://www.answers.com/topic/ ignaz-semmelweis (Accedido 15 de enero 2007).

3.- Nuland S. The Doctor`s plague: germs, childbed fever, and the strange story of Ignác Semmelweis, New York, Atlas Books/ Norton, 2003.
4.- Lee R, Chien A. Semmelweis. Med Hist 2005; 48: 616-8.

5.- Carter C K. Ignaz Semmelweis, Carl Mayrhofer, and the rise of the germ theory. Med Hist 1985; 29: 33-43.

6.- Carter C K Semmelweis and his predecessors Med Hist 1981; 25: 52-72.

7.- Bauer J. The tragic fate of Ignaz Phillip Semmelweis. Calif Med 1962; 48: 264-6

8.- Dunn P M. Ignaz Semmelweis of Budapest and the prevention of puerperal fever Arch Dis Child Fetal Neonatal Ed 2005;
90: 345-8.

9.- Raju T N. Ignác Semmelweis and the etiology of fetal and neonatal sepsis. J Perinatol 1999; 19: 307-10.

10.- Tan S Y, Brown J. Ignác Philipp Semmelweis (1818-1865): handwashing saves lives. Singapore Med J 2006; 47: 6-7.

11.- Gillies D. Hempelian and Kuhnian approaches in the philosophy of medicine: the Swemmelweis case. Studies in History and Philosophy of Biological and Biomedical Sciences 2005; 36: 159-81. 